\title{
Triple Phase Multidetector Computed Tomography of Hepatic Masses with Cytopathological Correlation
}

\author{
SAKSHI TOMAR ${ }^{1}$, MAMTA GOYAL $^{2}$, DN AWASTHY ${ }^{3}$, SHAILENDRA RAGHUVANSHI ${ }^{4}$
}

\section{ABSTRACT}

Introduction: Lesions of the liver may be benign or malignant and they can arise from hepatocytes, biliary epithelium, mesenchymal tissue or metastases from extra hepatic tissue. Along with Ultrasonography (USG), triple phase Multidetector Computed Tomography (MDCT) scan is a good non-invasive tool in characterising and differentiating benign from malignant liver lesions.

Aim: To characterise various hepatic masses with the help of triple phase MDCT scan and to correlate them with histopathological/ cytopathological findings.

Materials and Methods: By convenient sampling, in this observational study, 55 patients with primary diagnosis of hepatic masses on the basis of USG, were recruited and their triphasic MDCT scan findings were evaluated and later correlated with histopathology. SPSS version 22 and electronic Microsoft Excel spreadsheets were used. Categorical data has been represented as frequency (number) and proportions (percentages), continuous data as Mean \pm Standard Deviation (SD). ANOVA and Chi-square tests were used. Agreement between different diagnostic modalities was made using Kappa-statistic. The confidence level was kept at 95\%, hence a p-value $<0.05$ was considered as statistically significant.

Results: Among 55 patients, 4 (7.27\%) were benign and 51 (92.73\%) were malignant cases detected with the help of USG and same was verified on the basis of triphasic CT assessment. On histopathology, 50 (90.91\%) were identified as malignant lesion while $5(9.09 \%)$ cases were diagnosed benign lesions. Thus, triphasic CT scan has a sensitivity of $100 \%$, specificity of $80 \%$, Positive Predictive Value (PPV) of $98.04 \%$, Negative Predictive Value (NPV) of $100 \%$ and diagnostic accuracy of $98.18 \%$ in differentiating benign liver lesions from malignant liver lesions.

Conclusion: Early and accurate diagnosis of liver lesions is the foremost requirement in treatment. Triple phase MDCT scan along with USG can solve this and recommended in every suspected hepatic mass.

\section{INTRODUCTION}

Liver is an important constituent of the digestive tract to maintain body's metabolic homeostasis. Due to its major function of detoxification of body and its rich blood supply by hepatic artery and portal vein, it becomes prone to various diseases. Liver cancer is the sixth most common type of cancer in terms of incidence and third in terms of cancer related mortality worldwide [1]. The accurate and reliable determination of the nature of the liver mass is important to ensure that malignant lesions are diagnosed correctly. USG is an imaging technique that can provide anatomical and functional images with high resolution [2]. Emphasis has shifted on more advanced and more precise imaging techniques such as Computed Tomography (CT) and Magnetic Resonance Imaging (MRI) [3]. Multi-slice (multidetector-row) CT (MDCT) has a four-row configuration of detectors, sub-second gantry rotation time, and overcomes the limitations of single-slice CT scanners, especially in terms of scanning time and limited z-axis resolution [4]. One of the advantages of computed tomography is that it out-performs USG and MRI for evaluating the extra-hepatic abdomen [5]. With this background this study was conducted to evaluate triphasic MDCT in characterisation of hepatic masses and to correlate with histopathological/cytopathological diagnosis.

\section{MATERIALS AND METHODS}

This prospective, observational study was conducted in radiodiagnosis department of tertiary care teaching hospital over a period of 18 months from January 2016 to July 2017. Informed written consent was taken from patients about the participation in present study. Ethical clearance was granted by institute ethical committee [SRHU/HIMS/ETHICS/50].

\section{Sample Size Calculation}

In a recent study El-Sayed EE et al., reported the sensitivity of MDCT in detection of Hepatocellular Carcinomas (HCC) to be 90.3\% [6]. In present study we also targeted a similar sensitivity of HCC in a clinically suspicious population. The sample size had been calculated using the modification of a formula suggested by Snedecor GW et al., [7] to prove the hypothesis:

$$
\mathrm{n}=\left\{\mathrm{C} 2 \frac{p(1-p)}{e^{2}}\right\} / \text { Prevalence }
$$

Where ' $p$ ' is taken as the targeted sensitivity (90\% or 0.9 ), C is a constant at a certain confidence level (its value at $90 \%$ confidence limit and $80 \%$ power is 1.72). ' $e$ ' is the error allowance which is taken as 10\% (0.10) and prevalence of malignancy has been taken as $50 \%(0.5)$ in a screened population. Now placing these values in above equation we get 53.3. After adding for a contingency provision of $4 \%$ we get the sample size of 55 .

\section{Study Protocol}

All the patients with clinical diagnosis of hepatic mass underwent liver function test, serum creatinine and ultrasonographic assessment. Total 55 patients with primary USG diagnosis of hepatic mass were included for the study. Patients with USG features suggestive of abscess, hydatid cyst, simple cyst of liver and patients with metallic implant were not enrolled in the study.

All examinations were done on 64 slice MDCT. Opacification of digestive tract was achieved by oral administration of diluted $40 \mathrm{~mL}$ of ionic contrast in 2 liters of water. The patient was subjected to spiral CT scan and non-contrast $8 \mathrm{~mm}$ contiguous axial sections were taken from the level of domes of diaphragm up to the level of third lumbar vertebra. 
For an average adult patient nonionic contrast $100 \mathrm{~mL}$, of an ionic concentration of $320 \mathrm{mg} \mathrm{l} / \mathrm{mL}$ was injected automatically at the rate of $3-5 \mathrm{~mL} / \mathrm{second}$ with 325 psi intravenously. When contrast reached a $\mathrm{HU}$ value of 100 in the aorta, the arterial phase spiral images were obtained. After 25 seconds, the portal venous phase spiral images were obtained and delayed phase images were obtained after 6-10 minutes. Further imaging was done, if required as per the enhancing pattern of liver masses. All of these patients underwent either fine needle aspiration biopsy or core needle biopsy. Cytohistopathological findings were correlated with imaging findings.

\section{STATISTICAL ANALYSIS}

All relevant details including history, general clinical examination findings, radiological findings and cytopathological interpretations were recorded in case reporting form. A database was constituted using SPSS version 22 and electronic Microsoft Excel spreadsheets to store and manage the collected data. Categorical data has been represented as frequency (number) and proportions (percentages). Continuous data has been presented as Mean \pm Standard deviation (SD). For the analysis of data, ANOVA and Chi-square tests were used. Agreement between different diagnostic modalities was made using Kappa-statistic. The diagnostic efficacy of triphasic MDCT was expressed in terms of sensitivity, specificity, PPV, NPV and accuracy. The confidence level of the study was kept at $95 \%$, hence a $p$-value $<0.05$ was considered as statistically significant.

\section{RESULTS}

Total 55 patients, of age 3 years to 85 years, were enrolled in this study with mean age of $57.53 \pm 15.02$ years [Table/Fig-1]. Maximum number of patients were aged $61-80$ years (43.64\%) followed by those aged 41-60 years. In present study 34(61.82\%) patients were males. Among presenting clinical symptoms, abdominal pain (90.91\%) was the most common; whereas in clinical signs pallor (80.00\%), icterus (45.45\%), and Lump RHC (18.18\%) were the top three signs among the patients [Table/Fig-2]. As per USG assessment 51/55 (92.73\%) cases were malignant and 4/55 (7.27\%) benign, including $3(5.45 \%)$ hemangiomas and 1 (1.82\%) adenoma.

\begin{tabular}{|c|c|c|c|}
\hline SN & Characteristic & No. of cases & Percentage \\
\hline \multirow{7}{*}{1.} & Age (in years) & & \\
\hline & $<20$ & 1 & 1.82 \\
\hline & $21-40$ & 6 & 10.91 \\
\hline & $41-60$ & 23 & 41.82 \\
\hline & $61-80$ & 24 & 43.64 \\
\hline & $>80$ & 1 & 1.82 \\
\hline & Mean Age $\pm S D$ (Range) in years & \multicolumn{2}{|c|}{$57.53 \pm 15.02(3-85)$} \\
\hline \multirow{3}{*}{2.} & Gender & & \\
\hline & Male & 34 & 61.82 \\
\hline & Female & 21 & 38.18 \\
\hline
\end{tabular}

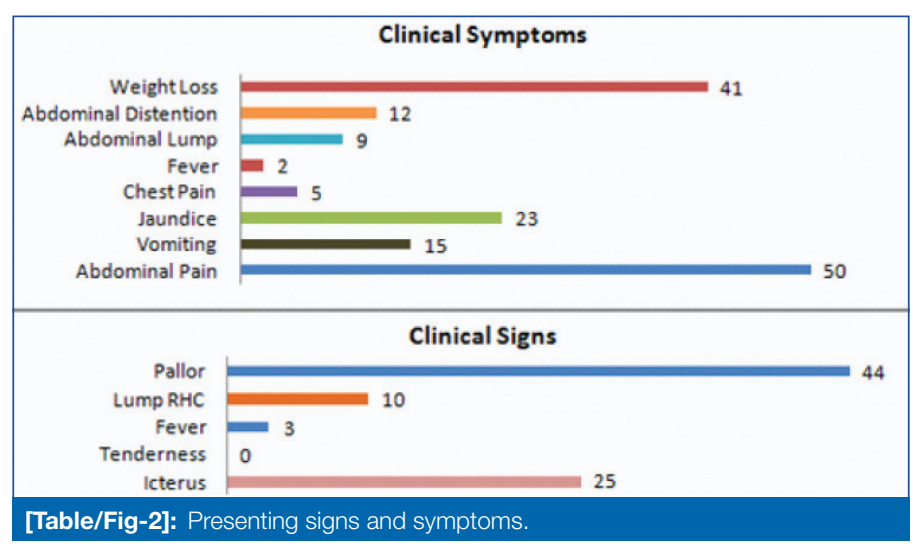

On triple phase MDCT assessment, 51 (92.73\%) hepatic masses were diagnosed as malignant and remaining 4 (7.27\%) were diagnosed as benign [Table/Fig-3]. Total 38 (69\%) hepatic masses were diagnosed as liver metastasis while 13 (23.64\%) were diagnosed as hepatocellular carcinoma [Table/Fig-4].

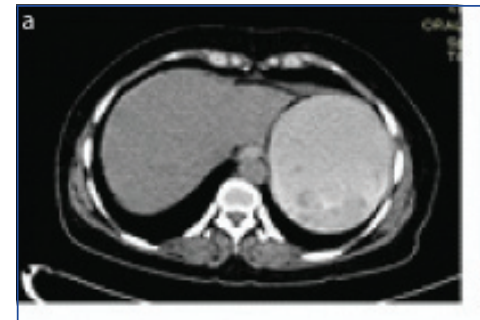

NCCT

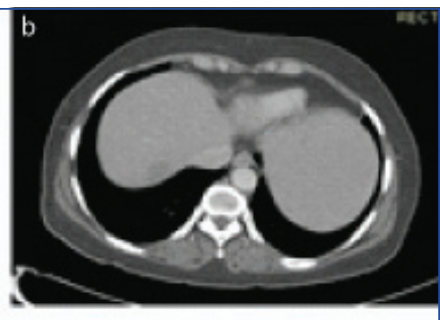

Arterial Phase

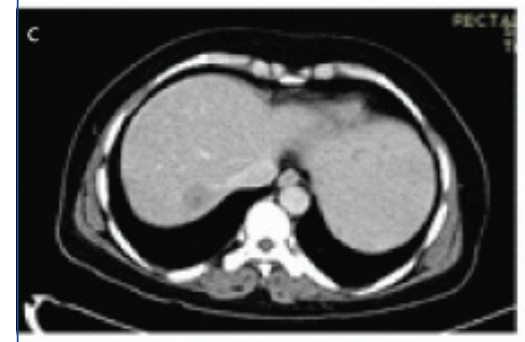

Portal Venous Phase

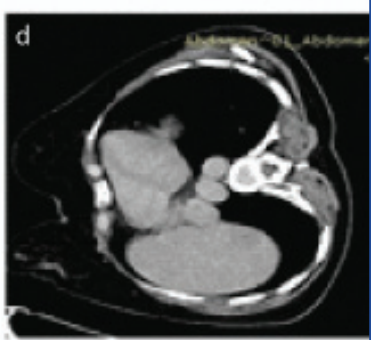

Delayed Phase [Table/Fig-3]: a) NCCT showing solitary hypodense lesion in right lobe of liver: $b, c, d) C E C T$ arterial, venous or delayed phase images showing no significant enhancement suggesting benign neoplasm (Adenoma).

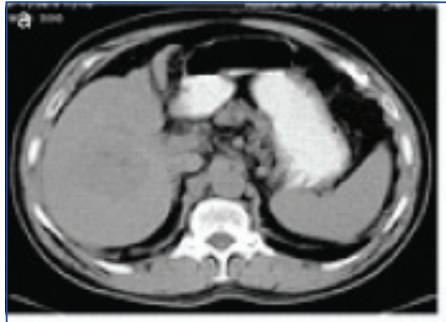

NCCT

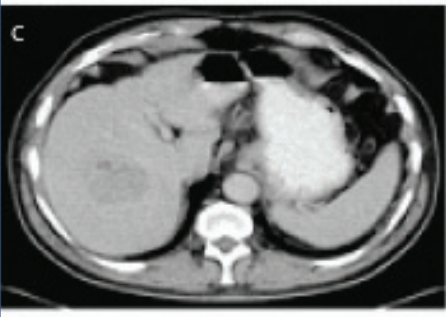

Venous Phase

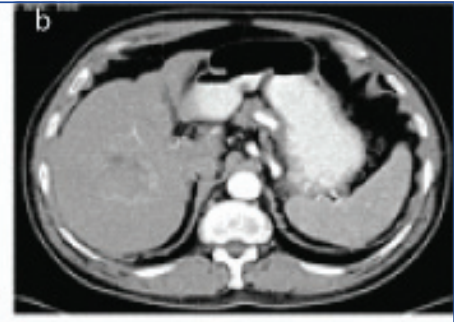

Arterial Phase

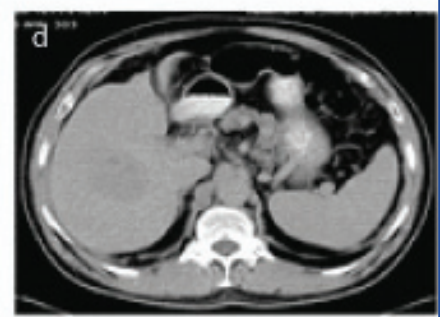

Delayed Phase
[Table/Fig-4]: a) NCCT showing solitary hypodense lesion in right lobe of liver: b,c,d) CECT showing heterogenous enhancement on arterial phase and rapid washout on portal venous and delayed phase images suggesting Malignant neoplasm (Hepatocellular carcinoma)

Among liver metastasis most common diagnosis was carcinoma gall bladder, in 14 (25.45\%) [Table/Fig-5]. There were 6 (10.9\%) cases of unknown primary, 2 (3.6\%) cases of carcinoma colon and carcinoma periampullary region and $1(1.82 \%)$ case each with carcinoma pancreas, carcinoma oesophagus, carcinoma stomach and renal cell carcinoma [Table/Fig-6].

Out of 55 hepatic masses on cytohistopathology 35 (63.64\%) were diagnosed as adenocarcinoma, 13 (23.64\%) hepatocellular carcinoma and one case each diagnosed as small cell carcinoma and malignant cells, respectively. There were 3 (5.45\%) cases diagnosed as adenoma and 2 (3.64\%) diagnosed as benign hepatocytes. On the basis of final diagnosis, a total of $50(90.91 \%)$ were identified as malignant while 5 (9.09\%) were benign hepatic mass. 


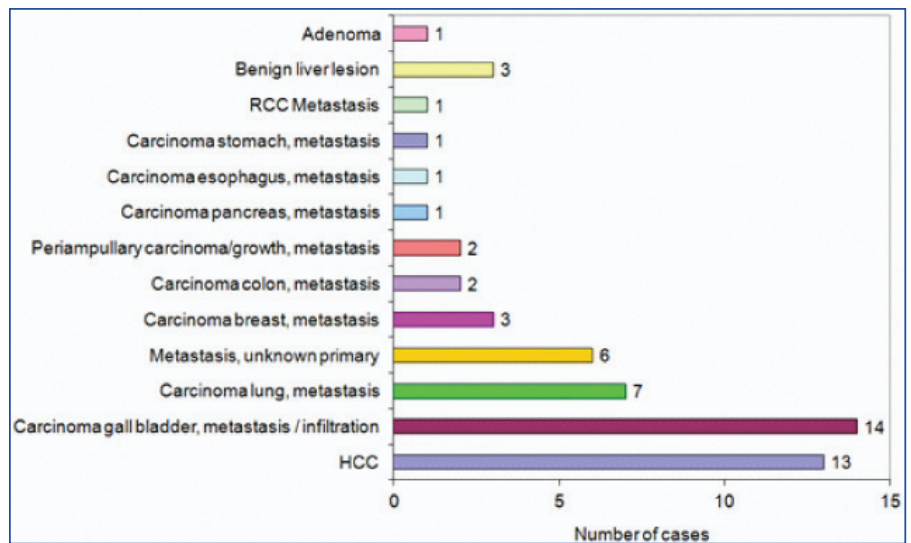

[Table/Fig-5]: Triphasic CT diagnosis of liver lesions.

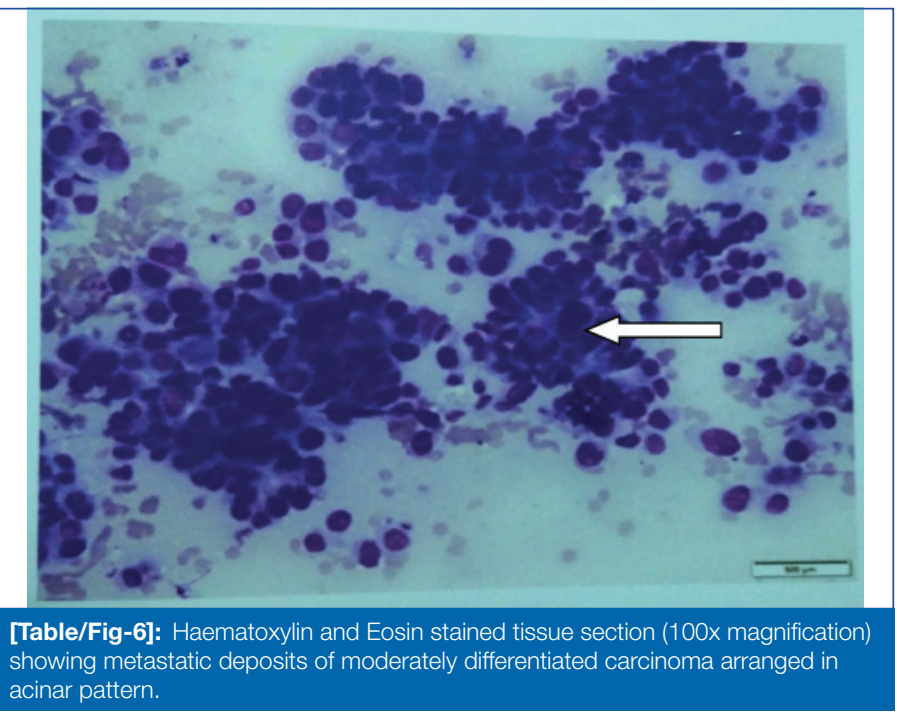

Except for 1 histopathologically proven case of benign mass which was wrongly interpreted as metastasis by triphasic CT, all the others were diagnosed perfectly. The level of agreement was excellent $(\kappa=0.962 ; p<0.001)$ [Table/Fig-7]. Triphasic CT detected 50 true positive, 1 false positive, none false negative and 4 true negative cases. Correspondingly, the sensitivity, specificity, positive and negative predictive values of triphasic CT for diagnosis of malignancy were $100 \%, 80 \%, 98.04 \%$ and $100 \%$ respectively. The method had an accuracy of $98.18 \%$ [Table/Fig-8].

\begin{tabular}{|c|c|c|c|c|}
\hline \multirow[b]{2}{*}{ SN } & \multirow[b]{2}{*}{ MDCT diagnosis } & \multicolumn{3}{|c|}{ Histocytopahological diagnosis } \\
\hline & & $\mathrm{HCC}$ & Metastasis & Benign \\
\hline 1. & $\mathrm{HCC}$ & 13 & 0 & 0 \\
\hline 2. & Metastasis & 0 & 37 & 1 \\
\hline 3. & Benign & 0 & 0 & 4 \\
\hline
\end{tabular}

\begin{tabular}{|c|c|c|c|c|}
\hline \multirow[b]{2}{*}{ SN } & \multirow{2}{*}{$\begin{array}{l}\text { Triphasic MDCT } \\
\text { diagnosis }\end{array}$} & \multicolumn{2}{|c|}{$\begin{array}{l}\text { Histocytopathological } \\
\text { diagnosis }\end{array}$} & \multirow[b]{2}{*}{ Total } \\
\hline & & Malignant & Benign & \\
\hline 1. & Malignant & 50 & 1 & 51 \\
\hline 2. & Benign & 0 & 4 & 4 \\
\hline \multicolumn{2}{|l|}{ Total } & 50 & 5 & 55 \\
\hline Sensitivity (\%) & Specificity (\%) & PPV (\%) & NPV (\%) & Accuracy (\%) \\
\hline 100 & 80 & 98.04 & 100 & 98.18 \\
\hline
\end{tabular}

\section{DISCUSSION}

In present study, we made an attempt to characterise 55 such hepatic masses and ended up in characterising 50 (90.9\%) of them as malignant and could diagnose only $5(9.1 \%)$ as benign.

The malignancy rates in different case series have shown to have a wide variability. Chung YE et al., in their study found a relatively lower malignancy rate at $26.0 \%$ [8]. On the other hand Lee HY et al. in their study found the malignancy rate as 34.1\% [9]. Böttcher $\mathrm{J}$ et al., in their study found $53.1 \%$ of lesions as malignant [10]. The evidence from neighboring country, Pakistan as shown by Hafeez $S$ et al., is similar to present study that found a high malignancy rate at $82.2 \%$ of patients and in $91.9 \%$ of total masses [11]. The other study from the region, the malignancy rate was much higher than these studies Chauhan $U$ et al. [12]. The reasons for the high malignancy rate in this series as well as in some of the other series from this region could be multiple - including poor diagnostic infrastructure, lack of adequate healthcare facilities, dietary habits, alcohol consumption, and treatment of early symptoms as simple gastrointestinal manifestations in the absence of a good primary healthcare structure. It must be kept in mind that most of the benign liver lesions have a high malignancy potential [13]. In the absence of adequate diagnostic facilities and detection at a delayed stage, the overall malignancy rates in our settings are relatively higher than that reported in series from outside. Another reason for high malignancy rate in present study was the strict exclusion criteria used by us. In present study, we excluded all the cases with USG features suggestive of abscess and cysts from inclusion, thus leading to an overall reduction of sampling universe, thus offering higher chances of malignancy detection.

The present study had a dominance of metastatic lesions. The proportion of metastatic masses among suspected liver masses has shown much variability. In their study, Hafeez $S$ et al., found metastatic lesions to be $37.5 \%$ of their entire sample [11]. Chauhan $U$ et al., found only $26.7 \%$ of their suspected masses to be metastatic [12]. However, Goel S et al., had $42.1 \%$ metastatic lesions in their study [14]. One of the reasons for high number of metastatic lesions in present study could be the high prevalence of carcinoma gall bladder in this belt. Interestingly, carcinoma gall bladder was the most common site responsible for metastasis to liver.

In present study, overall efficacy of triphasic MDCT in terms of sensitivity, specificity, PPV and NPV was 100\%, 80\%, 98\% and $100 \%$ respectively. The method had an accuracy of $98.2 \%$. Similar to present study, a high diagnostic efficacy of triphasic MDCT was reported by Hafeez S et al., who reported it to be $100 \%$ sensitive and $80 \%$ specific [11]. In their study the PPV was $94.6 \%$ and NPV was $100 \%$. They found triphastic MDCT to be $95.6 \%$ accurate.

The superiority of MDCT in detection of malignancy could be attributable to the ability of different phases based on vascular nature of masses. However, owing to less number of benign cases, only 1 misdiagnosis in the present study and 2 misdiagnosis in the study by Hafeez $S$ et al., led to loss of specificity by $20 \%$ [11]. In another study, that had only 4 benign cases as compared to 31 malignant cases, the sensitivity of MDCT was reported to be $90.3 \%$, however, owing to 2 misdiagnoses, the specificity was dropped down to $50 \%$ [14]. In the study which had adequate number of both benign as well as malignant cases, Goel $\mathrm{S}$ et al., found the sensitivity and specificity of triphasic MDCT to be $96.2 \%$ and $100 \%$, respectively [14]. Sinha R and Khatri KA, also reported the sensitivity of triphasic MDCT to be $97.6 \%$ for detection of metastasis [15]. In a recent study conducted by Jain $\mathrm{S}$ et al., overall sensitivity and specificity of MDCT for malignant lesions of liver was $83.3 \%$ and $97.2 \%$ [16].

\section{LIMITATION}

Limitation of present study is strict inclusion criteria. Owing to this limitation, this study was mainly restricted to description of malignant cases only. Further studies on larger size with slightly relaxed criteria of inclusion are recommended in order to get descriptive account of benign lesions. 


\section{CONCLUSION}

The findings of present study showed that triphasic MDCT is a useful modality for diagnosis and characterisation of liver masses, as it provides plentiful information from extra-abdomen primaries and hence is useful in situations where the liver lesions are not caused by a primary lesion and are metastatic in nature. However, less number of benign cases is a big barrier that needs to be surpassed for exact evaluation of usefulness of this modality. As a matter of fact, being more sensitive as well as specific, its use following a USG assessment seems to be a viable choice.

\section{REFERENCES}

[1] Ferlay J, Shin HR, Bray F, Forman D, Mathers C, Parkin DM. Estimates of worldwide burden of cancer in 2008: GLOBOCAN 2008. Int J Cancer. 2010;127:2893-917.

[2] McDicken W. Diagnostic ultrasound, physical principles and use of instruments, $3^{\text {rd }}$ edn. 1991, Churchill Livingstone, Edinburgh.

[3] Marrero JA, Ahn J, Rajender Reddy K; American College of Gastroenterology. ACG clinical guideline: The diagnosis and management of focal liver lesions. Am J Gastroenterol. 2014;109(9):1328-47.

[4] $\mathrm{Hu} \mathrm{H}, \mathrm{He} \mathrm{HD}$, Foley WD, Fox SH. Four multidetector-row helical CT: Image quality and volume coverage speed. Radiology. 2000;215:55-62.

[5] Hopper KD, Singapuri K, Finkel A. Body CT and oncologic imaging. Radiology. 2000;215:27-40.

[6] Elsayed EE, Koryem EM, Mohammed SA. Multidetector computed tomography in the detection of hepatocellular carcinomas meeting the Milan criteria before liver transplantation. Menoufia Med J. 2016;29:291-96.
[7] Snedecor GW, Cochran WG. Statistical Methods. $8^{\text {th }}$ Ed. Ames: lowa State Press, 1989.

[8] Chung YE, Kim MJ, Kim YE, Park MS, Choi JY, Kim KW. Characterization of incidental liver lesions: Comparison of multidetector CT versus Gd-EOB-DTPAenhanced MR imaging. PLoS One. 2013;8(6):e66141.

[9] Lee HY, Lee JM, Kim SH, Shin KS, Lee JY, Han JK, et al. Detection and characterization of focal hepatic lesions: Comparative study of MDCT and gadobenatedimeglumine-enhanced MR imaging. Clin Imaging. 2008;32(4):287-95.

[10] Böttcher J, Hansch A, Pfeil A, Schmidt P, Malich A, Schneeweiss A, et al. Detection and classification of different liver lesions: Comparison of Gd-EOBDTPA-enhanced MRI versus multiphasic spiral CT in a clinical single centre investigation. Eur J Radiol. 2013;82(11):1860-69.

[11] Hafeez S, Alam MS, Sajjad Z, Khan ZA, Akhter W, Mubarak F. Triphasic computed tomography (CT) scan in focal tumoral liver lesions. Pak Med Assoc. 2011;61(6):571-75.

[12] Chauhan U, Solanki RS, Udiya AK, Shetty GS, Narula MK. Triple phase computed tomography in hepatic masses. J Med Thesis. 2015;3(1):23-30.

[13] Elsayes KM, Narra VR, Yin Y, Mukundan G, Lammle M, Brown JJ. Focal hepatic lesions: diagnostic value of enhancement pattern approach with contrast enhanced 3D gradient echo MR imaging. RadioGraphics. 2005;25:1299-320.

[14] Goel S, Chowdhury V, Singh S, Puri AS, Sakuja P. Role of acoustic radiation force impulse elastography and triphasic computed tomography in the evaluation of focal liver lesions. Tropical Gastroenterology. 2016;37(3):191-202.

[15] Sinha R, Khatri KA. An observational study on the role of multidetector computed tomography in the evaluation of focal liver lesions. Int J Curr Med Appl Sci. 2017;14(2):90-95.

[16] Jain S, Khanduri S, Shah JK, Yadav P, Krishnam A. Role of MDCT in detection and characterization of focal liver lesions. Journal of Clinical and Diagnostic Research. 2019;13(5):TC01-TC05.

\section{PARTICULARS OF CONTRIBUTORS:}

1. Senior Resident, Department of Radiodiagnosis, Swami Rama Himalayan University, Dehradun, Uttarakhand, India.

2. Associate Professor, Department of Radiodiagnosis, Swami Rama Himalayan University, Dehradun, Uttarakhand, India.

3. Professor, Department of Radiodiagnosis, Swami Rama Himalayan University, Dehradun, Uttarakhand, India.

4. Professor, Department of Radiodiagnosis, Swami Rama Himalayan University, Dehradun, Uttarakhand, India.

\section{NAME, ADDRESS, E-MAIL ID OF THE CORRESPONDING AUTHOR:}

Dr. Mamta Goyal,

Associate Professor, Department of Radiodiagnosis, HIMS, Swami Rama Himalayan University, Jollygrant,

Dehradun-248016, Uttarakhand, India.

E-mail: drmamta2712@rediffmail.com

Date of Submission: Aug 07, 2019

Date of Peer Review: Aug 30, 2019

Date of Acceptance: Sep 20, 2019

Date of Publishing: Oct 01, 2019 